Théologiques

Théologiques

\title{
Conception et représentation fondamentales de l'esprit : une mise au point
}

\section{Richard Bodéüs}

Volume 2, numéro 2, octobre 1994

L'esprit

URI : https://id.erudit.org/iderudit/602404ar

DOI : https://doi.org/10.7202/602404ar

Aller au sommaire du numéro

Éditeur(s)

Faculté de théologie de l'Université de Montréal

ISSN

1188-7109 (imprimé)

1492-1413 (numérique)

Découvrir la revue

Citer cet article

Bodéüs, R. (1994). Conception et représentation fondamentales de l'esprit : une mise au point. Théologiques, 2(2), 7-20. https://doi.org/10.7202/602404ar
Résumé de l'article

Contre certaines opinions reçues, on s'efforce de montrer que l'opposition du corporel et de l'incorporel par laquelle l'« esprit » des traditions grecques et l'" esprit » de la tradition judéo-chrétienne semblent se distinguer l'un de l'autre, est une opposition secondaire, qui masque l'idée essentielle, commune aux représentations de l'esprit dans les deux traditions. Aux preuves apportées en faveur de cette idée essentielle, on ajoute un bref examen des conséquences de son ignorance, spécialement pour l'herméneutique du Nouveau Testament. 


\title{
Conception et représentation fondamentales de l'esprit : une mise au point
}

\author{
Richard BODËÜS \\ Département de philosophie \\ Université de Montréal
}

\section{RÉSUMÉ}

Contre certaines opinions reçues, on s'efforce de montrer que l'opposition du corporel et de l'incorporel par laquelle l' * esprit * des traditions grecques et l' * esprit * de la tradition judéo-chrétienne semblent se distinguer l'un de l'autre, est une opposition secondaire, qui masque l'idée essentielle, commune aux représentations de l'esprit dans les deux traditions. Aux preuves apportées en faveur de cette idée essentielle, on ajoute un bref examen des conséquences de son ignorance, spécialement pour l'herméneutique du Nouveau Testament.

Les théologiens qui m'ont demandé, comme historien de la philosophie, une contribution à ce numéro spécial, n'ignorent pas l'importance des considérations que la pensée grecque, dès avant l'ère chrétienne, avait développées sur l'«esprit». Le bilan de ces considérations a été dressé depuis longtemps et confronté à la pensée chrétienne dans une synthèse documentée, parue il y a près de quarante ans ${ }^{1}$. Je ne rendrais donc aucun

\section{1}

G. VERBEKE, L'évolution de la doctrine du pneuma du stoicisme à S. Augustin. Paris-Louvain, Desclée de Brouwer, 1945. La documentation de cet ouvrage devrair évidemment être mise à jour, mais elle reste impressionnante. Son point faible est ailleurs, dans l'hypothèse même de l'évolurion d'une doctrine du pneuma depuis la période hellénistique jusqu'au seuil du Moyen Âge. En fait, il y a, dans la seule tradition grecque, plusieurs doctrines du pneuma, qui ont, pour commune origine, des préoccupations médicales (voir, plus loin, n.21 et sqq.); l'une des principales est 
service à la recherche actuelle, si je ne prenais ici la liberté de montrer, dans la limite de mes moyens et de l'espace qui m'est alloué, en quoi cette synthèse me paraît insatisfaisante, lorsqu'elle prétend porter au crédit du christianisme d'avoir fourni à la pensée occidentale une conception «spiritualisante» de l'esprit ${ }^{2}$.

Pareille thèse me semble historiquement insoutenable, car, on le verra, c'est plus sûrement à la collusion du christianisme et de la philosophie grecque que l'on doit l'avènement d'une conception non matérialisante de l'esprit. Mais les droits de l'histoire sur ce détail seraient sans importance, n'était-ce qu'en raison de ce détail, la thèse à corriger passe visi-

intégrée à la physiologie des naturalistes de l'école d'Aristote (voir, à ce sujet, W. JAEGER, «Das pneuma im Lykeion *, Hermes 48 (1913) 29-74, reproduit dans Scripta minora. T. I, Rome, Edizioni di Storia e litteratura, 1960, p. 57-102; Diokles von Karystos. Die griechische Medizin und die Schule des Aristoteles. Berlin, de Gruyter, 1938 ; et Aristotle. Fundamentals of the History of His Development. Translated by R. Robinson, $2^{\mathrm{e}}$ éd., Oxford, Clarendon Press, [1934] 1967, Appendix I, p. 407-425); elle se retrouve jusque chez Galien ( $\mathrm{II}^{\mathrm{e}} \mathrm{s}$. de notre ère) et sans doute audelà ; une autre doctrine, parallèle, est intégtée à la philosophie naturelle (et, par elle, à la théologie) des Stoïciens : non seulement elle est en conflit avec sa rivale (cf. le dernier chapitre du Peri pneumatos anonyme du Corpus Aristotelicum, 485 a-486 b), mais elle présente, dès avant l'ère chrétienne, de sérieuses nuances d'un penseur à l'autre (Zénon, Cléanthe, etc. : voir, plus loin, sur le cas de Posidonios : n. 8 et 9). Il n'y a aucun sens à présenter la (?) ou les doctrines chrétiennes du pneuma, qui s'expriment dans le Nouveau Testament et qui s'inspirent fondamentalement de doctrines hébraïques, comme l'évolution de l'une de ces doctrines grecques contemporaines, ni d'une doctrine grecque en général; tout au plus, peut-on les comparer. Et encore, dans cetre comparaison, doit-on se garder de lire le Nouveau Testament (comme le fait Verbeke, p. 390-409), à la lumière des idées sur le pneuma que les penseurs chrétiens auront acquises ultérieurement ( $\mathrm{du} \mathrm{I}^{\mathrm{er}}$ au $\mathrm{V}^{\mathrm{e}}$ siècles) pour s'être nourris de philosophie grecque.

2 En gros, Verbeke fait observer (1) que les anciens Grecs en général avaient une conception matérialiste du pneuma (ils en parlent comme d'un corps) et (2) qu'à cette conception succède assez brusquement une conception chrétienne à contenu spiritualiste, laquelle finit par triompher. En réalité, l'observation (1) est celle d'un truisme; quant à l'observation (2), sur laquelle il étaie sa conclusion d'un * apport original du christianisme dans le monde " (p.544), elle n'a probablement aucun sens pour la pensée chrétienne des origines et, pour la pensée ultérieure, comme pour nous, c'est l'expression d'un pléonasme. De plus, il n'y a aucune *évolution * de l'idée du pneuma: nous parlons encore de l'«esprit de sel *, pour désigner, comme les anciens Grecs, un corps bien précis (l'acide chlorhydrique) et de l'«Esprit saint * pour désigner, que je sache, autre chose que l'incorporéité de l'une des trois personnes divines. 
blement à côté de l'essentiel quand il s'agit de confronter l'une à l'autre la tradition strictement grecque et la tradition strictement chrétienne sur le sujet. Au demeurant, devoir se résoudre à parler aujourd'hui d'une conception spiritualisante de l'esprit, sans craindre le pléonasme, c'est attester que l'essentiel n'a pas été vu.

Il est pourtant facile d'y voir clair. Partons, pour être bref, de l'usage des mots, qui est le nôtre. Le mot "esprit", dans l'usage de la langue française, même technique, renvoie toujours à un objet conçu comme étant d'une subtilité insaisissable ou très difficilement saisissable, objet mis en contraste, le plus souvent de façon explicite, avec quelque réalité brute ou concrète ou solide et qui se laisse pour ainsi dire toucher. Il est aisé de le montrer.

Les philosophes opposent l'esprit à la matière, au corps, à la nature, qui se laissent mesurer avec précision à l'aide d'instruments, ou encore à la sensibilité, qui se laisse éprouver, voire expérimenter. Et, lorsqu'ils s'efforcent de réduire l'immatériel, l'incorporel, le surnaturel ou le mental à ce qui ne l'est pas, c'est encore pour réserver à l'esprit ce qu'il y a de plus sublime et de plus subtil dans l'ordre de la matière ou de la sensibilité corporelle: c'est l'esprit selon Helvetius. Je ne rappellerai pas le langage des théologiens évidemment, qui opposent l'esprit à la chair, au monde périssable, et l'associent traditionnellement à notre âme, aux anges, aux démons, à Dieu lui-même. Les juristes, surtout depuis Montesquieu, se réclament de l'esprit des lois, subtilité redoutable qu'ils opposent à la grossièreté de la lettre, commodément lisible, dans un contraste sécularisé qui ne doit plus rien à celui de l'apôtre Paul (cf. $R m$ 2,29; etc.), mais le démarque de très loin. Les anciens physiologistes parlaient, eux, comme Descartes, des esprits animaux, secret d'une vie machinale, cachée sous l'épaisseur des carcasses. Et les anciens alchimistes nous ont légué leur esprit de sel, leur esprit de vin, de bois, de souffre, de vitriol, quintessences vaporeuses, distillées de la masse des corps bruts. On pourrait multiplier les exemples.

L'origine de tous ces usages, au fond convergents, est au bout d'une longue histoire, qui est aussi celle du mot "esprit" lui-même, issu du latin spiritus. Or celui-ci, on le sait parfaitement, servait à traduire, dès l'Antiquité, le grec pneuma dans une double tradition, celle des auteurs grecs eux-mêmes, savants ou hommes de lettres, et celle des auteurs bibliques, de la version des Septante ou du Nouveau Testament, qui s'en servaient pour rendre l'ancien hébreux rû'ah. Au départ, en effet, les termes grecs et 
hébreux avaient à peu près le même sens: "souffle, coup de vent... " ${ }^{3}$. L'idée élémentaire de l'insaisissable était déjà là, dans la représentation $\mathrm{du}$ vent qui souffle, s'insinue subtilement, bouscule, qu'on entend mais qu'on ne voit pas, et dans la représentation du souffle venteux qui soutient la vie animale, porte nos paroles, mais sur lequel nul n'a de prise.

Entre ces représentations vulgaires, très anciennes, et les spéculations philosophiques ou savantes, d'hier ou d'aujourd'hui, la distance n'est pas assez grande pour que l'analogie ne se remarque pas. Dans la dualité, inhérente à l'être, du matériel et de l'immatériel ou du corporel et de l'incorporel, l'esprit n'entre pas sans désigner la part de l'être qui échappe, par sa subtilité, à l'ordre des êtres ordinaires ou à la dimension ordinaire des êtres. De la même façon, dans la dualité, inhérente aux corps, de l'éthéré et du consistant, l'esprit dont parlaient les anciens chimistes n'entre pas sans signifier la part du corporel qui échappe, par sa subtilité, aux substances ordinaires ou à l'état ordinaire des substances, organiques ou inorganiques.

La seconde de ces dualités, pour qui s'arrête aux apparences, laisse croire à une conception "matérialisante" de l'esprit, qui semble constante dans la tradition des physiologues, des médecins et des philosophes de l'Antiquité ${ }^{4}$. La première, au contraire, fait surgir paradoxalement une conception de l'esprit tenu pour immatériel. Mais cette différence et le paradoxe qu'elle soulève sont, au fond, d'une importance très secondaire, dès lors que l'essentiel de la signification accordée au terme "esprit" n'est probablement révélé ni dans l'une ni dans l'autre de ces dualités, mais dans ce que l'une et l'autre ont en commun, en visant la subtilité insaisissable que l'être, d'un côté, et le corporel, de l'autre, présentent à l'un de leurs pôles. Ce qui se trouvait déjà visé dans la représentation vulgaire du souffle et du vent.

Il apparaît, dans ces conditions, que le fameux dilemne esprit-matière soulevé dans la philosophie occidentale depuis le Moyen Âge, fond ou, pour mieux dire, confond deux sortes d'interrogations parfaitement distinctes, dont l'une concerne exclusivement l'être en général et l'autre, indifféremment, l'être en général et le corporel qu'il contient manifestement. La première de ces deux interrogations formule l'alternative du

3 Comparez $\mathrm{Hb} 1,7$ (citant Ps 104, 1) ou 2 Th 2, 8 avec [ARISTOTE], De mundo, 4, 394 b 8-12.

4 Même quand ces philosophes, comme les Stoïciens, cherchent ainsi à identifier l'âme à un pneumachaud (cf. DIOGENE-L AERCE, VII, 157). 
matériel (ou du corporel) et de l'immatériel (ou de l'incorporel); quant à la seconde, la seule en fait où intervient l'esprit, elle met en place l'alternative qu'on peut appeler du saisissable et de l'insaisissable ${ }^{5}$.

Je reviendrai tout à l'heure sur les conditions historiques où, semble-til, s'est opérée la fusion de ces deux sortes de problèmes. Il importe d'abord de montrer que ces deux problèmes sont rigoureusement distincts et qu'ils peuvent - l'histoire le montre - être envisagés strictement l'un sans l'autre.

Que l'alternative du matériel (corporel) et de l'immatériel (incorporel) puisse être posée sans la moindre référence à l'esprit, c'est ce que montrent, à l'évidence, les philosophies d'Aristote et de Platon. Ces philosophies, en effet, connaissent ce genre d'alternative et offrent, chacune à leur façon, un plaidoyer en faveur de l'existence de l'immatériel (incorporel), mais conçu en termes stricts d'intelligible ou d'intelligence (pures substances formelles): on chercherait en vain, dans pareil plaidoyer, les traces d'une préoccupation sérieuse envers une quelconque subtilité insaisissable dépassant l'ordre matériel (ou corporel). Il serait plus aisé de montrer que c'est le contraire; inutile d'insister ${ }^{6}$.

Par ailleurs, il est possible de trouver des exemples précis de pensées où, à l'inverse, l'alternative du spirituel (pneumatique) et du saisis-

Le concept d'« insaisissable», obtenu à partir de la représentation du souffle, me paraît pouvoir être interprété de plusieurs façons, car c'est une généralité assez vague, susceptible de plusieurs spécifications. On verra, dans la suite, que je l'entends, selon les cas, d'un point de vue épistémologique (ce qui échappe à la connaissance) et d'un point de vue plutôt moral et religieux (ce qui ne se laisse pas approprier).

6 Dans le néoplatonisme, il y a un inintelligible (l'Un), situé au-delà de l'être; mais le plus haut degré immatériel de l'être, c'est l'intelligence (ou l'acte d'intellection), que certains traducteurs de Plotin ont tort d'appeler «esprit». On me permettra de noter que les traducteurs des Évangiles ont également tort de traduire par «esprit » le même mot grec noûs, comme, par exemple, dans Lc 24,45: «il leur ouvrit l'esprit (noûn) à l'intelligence (synienai) des Écritures». Il faut lire : «il leur ouvrit l'intelligence à la compréhension des Écritures ». La traduction fâcheuse de noûs par «esprit » dans ce passage (et dans d'autres) est, hélas!, commune et a été adoptée dans le récent Catéchisme de l'Église Catholique, publié en 1992 par le Service des Éditions de la Conférence des Évêques catholiques du Canada, p. 36, $\mathrm{n}^{\circ} 108$. L'index de ce Catéchisme, au mot « Esprit » (p. 648), est inutilisable en vertu de cette confusion: il regroupe indistinctement et sans qu'il soit possible de les reconnaître les unes des autres, des occurrences où il est question de l'esprit (pneuma) et celles où il est question de l'intelligence (noûs). Sur l'opposition de l'esprit et de l'intelligence, voir le célèbre passage de 1 Co 14,14 . 
sable se trouve posée, de manière totalement indépendante ou dans la plus grande indifférence à l'égard de la distinction entre le matériel (corporel) et ce qui ne l'est pas. Le premier exemple d'une telle pensée est fourni clairement par la philosophie stoïcienne.

Les Stoïciens, on le sait, n'ignorent pas le dilemne du corporel et de l'incorporel, mais ils le règlent d'emblée par une sorte de postulat logique, en posant avant toute autre discussion philosophique que tout ce qui est vraiment est un corps ${ }^{7}$. C'est donc une fois liquidée et comme évacuée cette question préjudicielle que s'articule, chez eux, la distinction entre ce qui est subtilité insaisissable et ce qui ne l'est pas ${ }^{8}$. Point n'est besoin de rappeler, ici, cette distinction stö̈cienne entre les masses consistantes de l'Univers et l'esprit (pneuma) qui les anime, animant aussi, à titre de Dieu, le tout qu'elles constituent. Mais indiquons tout de même, la formule que l'on prête au très célèbre Posidonios ( $\mathrm{II}_{-}{ }_{-} \mathrm{I}^{\mathrm{er}}$ ss. avant notre ère) pour décrire cet esprit: «un souffle d'intelligence et igné, qui n'a pas de forme, mais se change en ce qu'il veut" (d'après Stobée, I, 58) 9 . L'intérêt de cette formule théologique n'est pas dans l'affirmation que Dieu serait intelligent, car cela va de soi, ou qu'il serait corporel, car cela aussi, pour le philosophe du Portique, va de soi (dès lors qu'on peut établir que Dieu est quelque chose plutôt que rien). L'intérêt de la formule est dans l'assignation à Dieu d'une nature subtile et insaisissable. La subtilité de cette nature est garantie par son identification à un souffle (pneuma). Que ce souffle soit igné, plutôt qu'éthéré, n'est pas, ici, de grande importance ${ }^{10}$, l'essentiel étant qu'il soit propre, par son inconsistance, à s'insinuer partout ${ }^{11}$. Quant à son caractère insaisissable, lié à sa

7 Cf.J.BRuNSCHwig, "La théorie stoícienne du genre suprême et l'ontologie platonicienne », dans Matter and Metaphysics. Foutth Symposium Hellenisticum,. (J. Barnes and M. Mignucci, éd.), Naples, Bibliopolis, 1988, p. 21.

8 Voir, à ce sujet, les textes réunis dans A.A. Long et D.N. SEDLEY, The Hellenistic Philosophers. T. II, Cambridge, Cambridge University Press, 1987, p. 271-277.

9 Ce texte, avec d'autres, a été considéré par Verbeke, qui pensait pouvoir conclure du dossier Posidonios (où le feu intelligent est plus subtil que les autres corps qu'il pénèrre), que ce philosophe " a préparé les voies à la spiritualisation * (Op. cit., p. 142)!

10 Ce point marque cependant une différence entre la conception stoicienne et la conception défendue par Aristote et ses disciples, pour qui le pneumaest de nature, non pas chaude, mais fraîche et peur ainsi tempérer la chaleur innée des corps vivants, garantissant leur isothermie (cf. ARISTOTE, De la Respiration, 20, 480 a 14).

11 Cette subtilité est notée, en grec, par un mot précis ( to leptomeron: cf.SVF, I, 102 [= DIOGENE-LAERCE, VII, 142]); il s'applique, chez Aristore, aux particules élémentaires de feu, dont Démocrite déjà faisait l'âme (Fr. A 101 D-K = AR., De l'âme, I, 2, 405 b 5 . 
subtilité, c'est ce qui fait l'objet de la plus expresse insistance. Non seulement le souffle divin n'a pas de forme (permanente ou consistante) qui se laisserait appréhender ou connaître ${ }^{12}$, mais cela même qui le rend perpétuellement insaisissable (dans son perpétuel changement) est commandé par sa propre volonté: lui seul a les secrets de son être. On voit ici à quel point, en conférant à Dieu la nature de l'esprit, le philosophe grec répond à une question étrangère à celle de savoir s'il est corporel ou non, et dans quel sens assez précis va la question à laquelle il s'efforce de répondre en affirmant que Dieu est d'une subtilité insaisissable: il s'agit de savoir s'il est de nature telle que nous puissions le concevoir et percer son secret, la réponse suggérée étant négative. On notera que, dans ce genre d'interrogation, l'esprit intervient avec, si l'on ose dire, un profil étonnamment proche de la représentation que le sentiment populaire se faisait du vent.

Les Stoïciens n'ignoraient pas, par ailleurs, le problème que soulève la possibilité de l'immatériel, mais, nous venons de le voir, ce n'est pas du tout à ce problème de l'existence ou de la nature de l'immatériel que se rattache, chez eux, la notion d'esprit, même sur le terrain de la théologie. Il est une autre forme historique de pensée, qui, comme celle des Stoïciens, pose l'esprit comme insaisissable dans certaines alternatives, mais avec plus d'indépendance encore par rapport à la question philosophique de l'immatérialité ou de l'incorporéité, qu'elle ignore. C'est la pensée des auteurs néotestamentaires, installés dans la tradition biblique.

On connaît la très célèbre formule johannique $(4,24)$ : «Dieu est esprit» (pneuma). On sait également que cette affirmation lapidaire, placée dans la bouche du Christ, est un élément de réponse que celui-ci fournit à la Samaritaine lui demandant s'il convient d'adorer Dieu dans le temple du Garizim ou celui de Jérusalem; et l'on sait que le sens général de cette réponse est que la question, au fond, n'importe pas, parce que Dieu n'est lié à aucun lieu ${ }^{13}$. De ce que, pour un philosophe grec, tout ce

13); il caractérise le corps particulier dont Épicure, lui aussi, faisait l'âme (Lettre à Hérodote, 63,4$)$ et que le médecin épicurien Asclépiade ( ${ }^{\mathrm{er}}$ siècle avant notre ère) appelait pneuma (d'après CcFLIUS AUR., Maladies aiguës, I, 113).

12 Malgré que la nature divine soit par ailleurs intelligence et raison (logos), ce n'est pas en sa qualité de pneumaqu'elle se laisse connaître (et connaît). En cette qualité, elle est, au contraire, partout et partout insaisissable.

13 Il est malaisé de donner le sens précis de la suite du passage («ceux qui l'adorent doivent le faire en esprit et vérité »). Mais il est certainement faux de penser que le 
qui n'est pas localisable est incorporel ${ }^{14}$, on pourrait en conclure que, dans la formule du Christ, l'identification de Dieu à l'esprit est une façon de dire son incorporéité. Je crains fort qu'il n'en soit strictement rien et que la formule ne tranche aucune question de ce genre, mais, dans le style de l'évangéliste, indique, au contraire, qu'il est en quelque sorte dans la volonté de Dieu d'échapper à toute tentative d'emprisonnement par quelque rite local que ce soit. Ce qui est, comme chez les Stoïciens, le ranger dans l'insaisissable, qu'aucune tentative humaine ne peut s'approprier en le fixant. Car c'est le même évangéliste qui affirme encore $(3,8)$ que "le pneumasouffle où il veut ", qu'on entend sa voix, mais qu'on ne sait d'où il vient, ni où il va, recourant ainsi à la représentation populaire du vent insaisissable et imprévisible pour suggérer l'avantage de ceux que l'esprit a fait renaître et à qui Dieu donne, en somme, un second souffle. Or si cette analogie a du sens, et elle en a, il faut comprendre que ce qui est dit du vent (pneuma) en 3,8, savoir qu'il souffle où il veut, peut aussi se dire d'une certaine façon de Dieu, qui, en 4, 24, est dit pneuma. Et, dans ces conditions, on obtient, à peu près la même formule que celle par laquelle Posidonios décrivait Dieu: il (se) fait ce qu'il veut. Chez l'évangéliste et le philosophe grec, l'esprit (pneuma) indique de manière appropriée la subtilité par où Dieu échappe, au fond, à tout effort pour être saisi.

Ces exemples simples, mais qu'on pourrait approfondir, montrent, me semble-t-il, comment, historiquement, les traditions anciennes distinguaient l'un de l'autre, l'antagonisme du matériel (corporel) et de l'immatériel (incorporel), qui concerne l'être en général, et l'antagonisme du saisissable et de l'insaisissable, que sert à poser l'esprit. Avant de poursuivre, on peut indiquer les conditions où ces deux antagonismes se sont fondus en un seul, jusqu'à créer l'opposition ambiguë matière-esprit, à laquelle nous sommes accoutumés.

Les conditions du phénomène sont complexes, mais on ne surprendra personne en avançant qu'elles ont été fournies par ce qu'on appelle

Christ plaide pour une adoration intérieure "par ce qu'il y a de plus intime dans l'homme: l'âme, intelligence et volonté » (VERBEKE, op. cit., p. 390). C'est probablement la rigidité des formalismes cultuels qui est mise en cause, sans plus: le reste est spéculation gratuite.

14 Le défaut de localisation, dans la tradition biblique (cf. $1 R 8,27$ ), est conçu autrement, comme «ce qui ne peut être contenu ». 
l' "hellénisation du christianisme" 15 , durant les premiers siècles de notre ère. Cette époque est le moment où la pensée nourrie des Écritures Saintes et gagnée jusqu'alors à l'idée d'un souffle divin insaisissable, est confrontée à la philosophie grecque et, de ce fait, pressée de s'interroger sur la matérialité d'un tel être divin. On a dit plus haut que, du côté des philosophes Stoïciens, la question préjudicielle était celle qui doit décider de la matérialité ou de l'immatérialité de l'être et que la pneumatologie qui fixe Dieu du côté de l'insaisissable venait chez eux en second lieu. Dans la pensée chrétienne, la question préjudicielle, au contraire, était traditionnellement celle, strictement religieuse, de la position de Dieu par rapport au monde et à l'homme, question que réglait d'emblée la Genèse, en opposant la subtilité insaisissable du souffle divin à l'épaisse brutalité du chaos $(1,2)$ et de la glaise adamique $(2,7)^{16}$. Dans ces conditions, quand la philosophie grecque invita la pensée chrétienne à se poser clairement la question de l'immatérialité de Dieu, cette question appela spontanément une réponse positive et le témoignage johannique, dont on a parlé, finit par être reçu comme une caution en ce sens. Ainsi, voit-on ultimement procéder Thomas d'Aquin, qui était pourtant averti de ce qu'est l'esprit «dans les choses corporelles»17: c'est le témoignage de Jean, 4, 43 (spiritus est Deus) qui se trouve produit, comme seule autorité, dans le sed contrade la question "Utrum Deus sit corpus?" (ST, Ia-Iae, qu. 3, art. 1). L'importance de la théologie chrétienne, dans les questions métaphysiques au Moyen Âge, suffit désormais à imposer l'esprit comme synonyme d'incorporel.

Il en est résulté des conséquences pour les questions à débattre, si l'on ose dire, en aval et en amont. En aval, depuis les Temps Modernes et jusqu'à nos jours, les questions philosophiques ont engagé l'esprit dans un dilemne où il s'oppose non seulement au saisissable, mais à la matière: «Au regard de tous les phénomènes matériels, l'esprit c'est l'autre. Autre

15 À ce sujet, voir M.C. BARTOLOMEI, Ellenizzazione del cristianismo. Linee di critica filosofica e teologica per una interpretazione del problema storico. L'Aquila-Rome, 1984; et notre compte-rendu de cet ouvrage dans RevPhillouv 84 (1986) 385-386.

16 Il est intéressant de noter que le premier de ces passages était encore invoqué au $\mathrm{XVII}$ siècle par Leibniz pour cautionner sa conception du mouvement éthéré en circulation autour de la masse terre-air-eau de notre globe (Hypothesis physica nova dans SSB, VII, 2, p. 225).

17 Cf. ST, la-lae, q. 36, art. 1, conclusio : « Nam nomen Spiritus in rebus corporeis impulsionem quamdam et motionem significare videtur; nam flatum et ventum spiritum nominamus . 
que tout ce qui peut être mesuré, observé, compté, vérifié ${ }^{18}$ ». Dès lors, tout se passe comme si la matière pouvait être réduite à l'épaisseur du saisissable, et comme si l'immatériel devait échapper à l'intelligible. Ce genre de conviction consacre, maintenant encore, le divorce malheureux entre une science "dure", conspuée pour ses certitudes massives, et une pensée "légère", non scientifique, décriée pour son inintelligibilité éthérée.

En amont, se trouvent notamment les questions que continue à soulever l'herméneutique des écrits néotestamentaires et qu'éclaire désormais une conception de l'esprit où s'efface l'idée de l'insaisissable au profit de l'idée d'immatérialité. Comment, en effet, ne pas voir désormais, dans l'affirmation banale que "le corps sans esprit (pneuma) est cadavre" $(J c 2,26)$, une affirmation qui vise à poser l'incorporéité de l'esprit? Comment, sinon en s'efforçant d'oublier la dualité du matériel et de l'immatériel, étrangère à ce passage, et en considérant qu'ici, tout comme dans les nombreux autres textes qui distinguent l'esprit et le corps ${ }^{19}$, c'est littéralement ou par métaphore, la subtilité insaisissable du souffle vital qui est en cause et qui s'oppose, non à la corporéité des philosophes, mais à la rigidité cadavérique du corps sans vie? Même la question de savoir ce qu'est un esprit (pneuma) en dehors du corps (vivant) est une question qui ne reçoit pas, dans le Nouveau Testament, une réponse allant dans le sens de l'incorporéité. Le passage décrivant la terreur des Apôtres devant

18 Cl.BRUaIRE, La force de l'esprit. Entretien avec Emmanuel Hirsch. Paris, Desclée de Brouwer, 1986, p. 16.

19 Cf. Mt 26,41; Mc 14,38; Ac 2, 17 (cf.Jo 3,1); Rm 8, 4.13; 1 Co 5, 5; 6, 19 ; 7,34; 12,$13 ; 2$ Co 7,$1 ; \mathrm{Ga}, 3,3 ; 4,29 ; 5,17 ; \mathrm{Ep} 4,4 ; 1 \mathrm{Th} 5,23 ; 1 \mathrm{P} 4,6 ; \ldots$ Les passages où l'incorporéité de l'esprit semble le mieux suggérée sont $L c$ 3, 22 : l'esprit saint descend " sous forme corporelle» (cf. In 1,32-33) et 1 Co 5, 3: « absent de corps, mais présent d'esprit ». Mais aucun de ces textes ne s'interroge, ni ne renseigne sur la nature divine de l'« esprit». Le premier signifie que l'ES devint visible, c'est-à-dire, qu'il fut visible que le Christ baptisé avait reçu de secrètes faveurs de la part de Dieu; et le second use d'une image pour dire que l'absence de quelqu'un ne doit rien changer à ce que serait sa présence. -Quant à la question du rapport délicat entre âme et esprit (sur l'intelligence, voir, plus haut, n.6), il ne se pose pas du tout dans les termes de l'anthropologie grecque dont nous avons hérité. Il serait, sinon, impossible de parler d'hommes "psychiques sans esprit» (cf. 1Co2,14;3,1;Jc 3,15). Quand l'esprit paraît mal se distinguer de l'âtme, comme principe de la vie individuelle (cf. $L c 1,47 ; R m$ $12,11)$, c'est qu'il désigne cet irréductible principe de la vie qui n'appartient pas à l'individu, mais à Dieu. Ce "point de départ " entre l'âme et l'esprit (dont parle $\mathrm{He}$ 4,12 ), marque précisément le partage entre le terrain de l'anthropologie, au sens grec et profane du terme, et celui de la théologie, quand elle parle de l'homme. 
l'apparition du Christ ressuscité (Lc 24,37: «il leur semblait voir un esprit» [pneuma]), contient cette parole apaisante du ressuscité : «palpezmoi et voyez: un esprit (pneuma) n'a ni chair, ni os, comme vous me voyez en avoir" $(24,39)$. Ce n'est pas l'insensibilité qui, rigoureusement, distingue l'esprit, lequel peut être vu, entendu,...; c'est son caractère inconsistant, insaisissable. Le texte n'enseigne pas, pour autant, la matérialité du pneuma, pas plus qu'il n'enseigne maladroitement son immatérialité. La question, en définitive, n'est pas là. Et c'est ce qu'il faut se dire, selon moi, sous peine de passer à côté de l'essentiel.

On ne peut sans inconvénient majeur, je crois, retourner à l'essentiel de la pneumatologie néotestamentaire, en utilisant, comme outil herméneutique, la dualité esprit-matière de la philosophie moderne, dont j'ai dit, il y a un instant, l'ambiguïté fâcheuse. Le peut-on en utilisant l'outil que présente la pneumatologie grecque antérieure au christianisme, celle des Stoïciens ou des physiologistes, médecins ou naturalistes, dont on sait qu'elle a spéculé sur la subtilité d'un souffle vital?

Comme la majorité des exégètes, je ne crois pas que la pneumatologie du Nouveau Testament, même paulinienne, s'explique par quelque source "païenne" que ce soit. Mais elle entretient, avec la pensée des philosophes et des naturalistes grecs, des analogies indéniables, dont Paul a peut-être eu le sentiment. Ainsi, lorsqu'il oppose la sagesse du siècle et celle qu'on reçoit de Dieu, en disant: "nous n'avons pas reçu le pneuma du monde (kosmos), mais le pneumaqui vient de Dieu " (1 Co 2,12). L'idée du «pneumade vérité que le monde (kosmos) ne peut recevoir» est aussi dans Jn 14, 17; mais celle, corrélative, du pneumacosmique, que Paul énonce, sonne décidément comme une idée stoïcienne ${ }^{20}$. Quoiqu'il en soit, les analogies sont là, même si elles ont échappé à l'Apôtre. Peut-on les exploiter avantageusement?

Je pense qu'elles ont l'avantage de fixer clairement deux perspectives où s'intègrent les doctrines pneumatologiques, tant du côté grec que du côté chrétien. Chez les Grecs, en effet, par-delà la diversité des doctrines, le souffle (pneumatique), qui est réputé avoir son siège dans le cœur le plus souvent ${ }^{21}$, est chargé d'expliquer deux fonctions principales du vivant: la

20 À comparer avec « les éléments du kosmos " de Col 2, 8 et le commentaire de ce dernier passage dans G. BABAGIO-R.FABRIS, Lettera ai Colossesidans Le lettere di Paolo. T. III, Rome, 1980 (ad locum).

21 Ce qui est aussi le cas dans $R m 5,5 ; 2$ Co 1,22 ; etc. - Du côté grec, la littérature médicale ancienne hésite entre le cœur et le cerveau (cf.[HIPPOCRATE], Maladie sacrée, 3-7 et 16; Aliments, 31; Chairs, 5; Cour, 6). Pour la circulation du pneumadans les 
première est une fonction cognitive, celle de la sensibilité et parfois de 1'intelligence 22 , et la seconde, la plus courante, est une fonction "cinétique", le souffle agissant comme outil à la fois dans la différenciation des parties du vivant embryonnaire et dans la motricité, chez l'animal complet ${ }^{23}$. Il est aisé de voir qu'aux deux perspectives ainsi définies, correspondent, en gros, deux puissances du souffle (pneumatique) que le Nouveau Testament place aussi dans le cœeur: ce sont, d'une part, celle qui permet de recevoir et dire la vérité, dont il vient d'être question et qui est liée à la prophétie ${ }^{24}$, et d'autre part, celle "vivifiante», qui produit, conserve ou rend la vie ${ }^{25}$. Ce genre de similitudes est peut-être intéressant, dans la mesure où il atteste que, de part et d'autre, du côté grec et du

veines ou artères, voir en particulier M.-P. DuMINIL, Le sang, les vaisseaux, le cœur dans la Collection hippocratique. Anatomie et physiologie. Paris, Société d'édition Les Belles Letrres, 1983, 255-272 et 301-305.

22 Pour l'intelligence, voir, outre les Stoïciens, [HIPP.], Cœur, 10 et Maladie sacrée, 16.

23 La différenciation des parties de l'embryon par le pneumaest déjà indiquée dans [HIPP.], Nature de l'enfant, 15, 17-20 (traité du début du IV siècle avant notre ère, vraisemblablement), qui fait aussi du souffle le moteur du sang (et du sperme, selon Aphorismes, V, 63). Le souffle inné a le même rôle de différenciation des parties chez Aristote (Génération des animaux, II, 6, 743 b 29 et sqq.); lequel cependant insiste sur sa fonction motrice (Parties des animaux, II, 16, $659 \mathrm{~b}$ 17-18). C'est chez Aristote que les deux rôles du pneumasont, pour la première fois, précisés dans une synthèse cohérente ( $\mathrm{cf}$. ci-dessus, n. 10). Le mouvement, fonction distinctive de l'animal, doit compter sur un souffle inné et sur un souffle respiré, pris à l'extérieur; le pneuma est ainsi l'instrument de l'âme proprement animale (cf. Mouvement des animaux, 9-10), car, dans le cœur, siège central de la sensibilité, il est également l'organe corporel de l'âme sensitive (cf. Génér. an., 744 a 1 et sqq.). - - Il est à remarquet qu'en dépit de son rôle dans l'organisation de la vie embryonnaire et de son association intime (comme outil) avec l'âme sensitive et motrice, qui caractérise la vie animale, le pneumadu naturaliste grec est seulement le ressort le plus subtil des mécanismes physiologiques: il n'est pas, proprement, une source de vie.

24 Cf. 1 Co 2,11. Les expressions «esprit de vérité $($ In 14,17) et «esprit de prophétie » $(A p 19,10)$ semblent, à cet égard, interchangeables, dans l'exacte mesure où la vérité chrétienne est touJnurs prononcée au nom de Dieu qui la souffle (cf. Mc 13, 11).

25 Cf. In 3,$6 ; 6,63 ; R m 8,16 ; 1$ Co 15,$45 ; 2$ Co 3,6; 1 P 3, 18; Ap 11, 11. Selon la même idée, la mort n'est pas simple « expiration " (cf. $M t 27,50$ ), mais * restitution d'un souffle qui, fondamentalement, ne nous appartient pas (cf.Jn 19, 30), mais appartient à Dieu (et repose «dans ses mains ": Lc 23,46). 
côté chrétien, l'on réfléchissait probablement sur la base d'une même représentation élémentaire du souffle. Mais l'observation des ressemblances ne conduit pas fort loin et masque de profondes différences ici et $1 \mathbf{a ̀}^{26}$.

En particulier, elles masquent combien la notion de subtilité insaisissable, que contient l'idée de pneuma, est entendue différemment de part et d'autre. Dans la tradition grecque, en effet, les penseurs qui font état de ce souffle pneumatique sont strictement avertis (depuis Platon) de la distinction du corporel et de l'incorporel et tous, pratiquement sans exception, reconnaissent dans le pneuma un corps subtil, c'est-à-dire un corps dont l'insaisissabilité est synonyme, rigoureusement, d'intangibilité. C'est pourquoi, lorsque, plus tard, le pneumasera tenu pour incorporel, l'inaccessible au sens du toucher deviendra, par une sorte de transfert, synonyme d'inaccessible à l'intelligence, et les philosophies de l'esprit se flatteront ainsi de préserver un épistémologiquement inconnaissable.

Dans la littérature chrétienne du Nouveau Testament, il en allait tout autrement. Étrangère, répétons-le, à la distinction du corporel et de l'incorporel, la pensée des auteurs chrétiens du Nouveau Testament entendait, de ce fait, l'insaisissable du pneumadans une pespective où n'entraient pas les questions épistémologiques de la connaissance (sensible ou intelligible). Le souffle intangible, sur lequel on échoue à mettre la main, renvoyait, pour eux, à ce sur quoi nous n'avons pas de prise (comme Dieu, la vie, etc.), un genre de force que nous ne commandons pas et qui, lorsqu'elle nous échoit, nous commande au contraire. Au fond, l'esprit (pneuma) est, dans l'homme ou hors de lui, ce qu'il ne maîtrise pas.

M'est avis que cette notion, quoique vague, est assez précise pour faire entrer dans une pensée sui generis des auteurs chrétiens, qu'oblitèrent les perspectives de la tradition grecque.

Ces considérations, si elles sont justes, n'invitent pas, cependant, à l'ignorance des traditions grecques pour l'exégèse herméneutique du Nouveau Testament. Elles inviteraient plutôt, au contraire, à prendre en

26 La remarque vaut singulièrement pour la distinction, qu'on retrouve dans les deux traditions, entre bon esprit et mauvais esprit, esprit pur (saint) et esprit impur (cf. He 1,$14 ; A p 4,5$ et 5,$6 ; M_{t} 12,43$; etc.). Les traités hippocratiques Des vents ( $V^{\mathbf{e}}$ siècle avant notre ère) et, en partie, Eaux, airs, lieux (même époque, chap.3-6) accordent une importance décisive à la pureté de l'air et le premier de ces traités fait des pneumata la cause unique de toutes les maladies. 
compte ces traditions, mais en ce qu'elles ont, elles aussi, de sui generiset qui n'est pas fondamentalement, on le voit, une conception matérialisante ou corporelle de l'esprit, mais une conception philosophique et épistémologiquement élaborée du corporel.

La pensée chrétienne, liée à cette herméneutique, peut reconnaître ainsi le prix qu'elle a dû payer pour asseoir, dans la pensée onto-théologique, l'immatérialité d'un Dieu-esprit: introduire, à l'avant-plan de l'ontologie, un épistémologiquement inconnaissable que la tradition grecque ignorait, et oublier, dans la théologie, l'humainement immaîtrisable que la pensée évangélique cultivait. 